\title{
Cloud Computing-Based Forensic Analysis for Collaborative Network Security Management System
}

\author{
Zhen Chen*, Fuye Han, Junwei Cao, Xin Jiang, and Shuo Chen
}

\begin{abstract}
Internet security problems remain a major challenge with many security concerns such as Internet worms, spam, and phishing attacks. Botnets, well-organized distributed network attacks, consist of a large number of bots that generate huge volumes of spam or launch Distributed Denial of Service (DDoS) attacks on victim hosts. New emerging botnet attacks degrade the status of Internet security further. To address these problems, a practical collaborative network security management system is proposed with an effective collaborative Unified Threat Management (UTM) and traffic probers. A distributed security overlay network with a centralized security center leverages a peer-to-peer communication protocol used in the UTMs collaborative module and connects them virtually to exchange network events and security rules. Security functions for the UTM are retrofitted to share security rules. In this paper, we propose a design and implementation of a cloud-based security center for network security forensic analysis. We propose using cloud storage to keep collected traffic data and then processing it with cloud computing platforms to find the malicious attacks. As a practical example, phishing attack forensic analysis is presented and the required computing and storage resources are evaluated based on real trace data. The cloudbased security center can instruct each collaborative UTM and prober to collect events and raw traffic, send them back for deep analysis, and generate new security rules. These new security rules are enforced by collaborative UTM and the feedback events of such rules are returned to the security center. By this type of close-loop control, the collaborative network security management system can identify and address new distributed attacks more quickly and effectively.
\end{abstract}

Key words: cloud computing; overlay network; collaborative network security system; computer forensics; antibotnet; anti-phishing; hadoop file system; eucalyptus; amazon web service

\section{Introduction and Background}

With the Internet playing an increasingly important role

- Zhen Chen and Junwei Cao are with Research Institute of Information Technology and Tsinghua National Laboratory for Information Science and Technology (TNList), Tsinghua University, Beijing 100084, China. E-mail: zhenchen@tsinghua.edu.cn; jcao@tsinghua.edu.cn.

- Fuye Han and Xin Jiang are with Department of Computer Science and Technology, Research Institute of Information Technology and Tsinghua National Laboratory for Information Science and Technology (TNList), Tsinghua University, Beijing 100084, China. E-mail: hanchao1107@gmail.com; jiangxin_thu@sina.cn.

- Shuo Chen is with Department of Automation, Research Institute of Information Technology and Tsinghua National Laboratory for Information Science and Technology (TNList), Tsinghua University, Beijing 100084, China. E-mail: chenatu2006@sina.com.

* To whom correspondence should be addressed. Manuscript received: 2012-12-15; accepted: 2013-01-15. as our information infrastructure, the e-business and e-pay sector is booming due to its convenience and benefits for users. However, Internet security remains a big challenge as there are many security threats. The underground economics based on Internet scams and frauds is also booming. Attackers increasingly initiate e-crime attacks and abuses ${ }^{[1-5]}$, such as spam, phishing attacks, and Internet worms. Firewalls, Intrusion Detection Systems (IDS), and Anti-Virus Gateway are now widely deployed in edge networks to protect end-systems from attack. When malicious attacks have fixed patterns, they can be easily identified by matching them to known threats ${ }^{[6-9]}$. However, sophisticated attacks are distributed over the Internet, have fewer characteristics, and evolve quickly. For example, a Distributed Denial of Service (DDoS) attack contains 
very few, if any, signature strings to identify.

Nowadays, DDoS attacks are likely to be launched by a large volume of bots - a botnet-controlled by a bot-master. The bots are commanded to create zombie machines and enlarge the botnet as well as to disseminate spam or to launch DDoS attacks on victim hosts. To countermeasure botnets, a secure overlay is proposed. To prevent distributed attacks, collaboration is required. Collaborative intrusion detection system is reviewed by researches in Ref. [10]. By collaborating, the network security system embraces scalability and teamwork and has a better overview of events in the whole network. A collaboration algorithm is presented to improve the alert events accuracy by aggregating information from different sources in Ref. [11]. A similar alert correlation algorithm ${ }^{[12]}$ was put forward, which is based on Distributed Hash Tables (DHT).

The Collaborative Network Security Management System (CNSMS $)^{[13]}$ aims to develop a new collaboration system to integrate a well deployed Unified Threat Management (UTM) such as NetSecu ${ }^{[14]}$. Such a distributed security overlay network coordinated with a centralized security center leverages a peer-to-peer communication protocol used in the UTMs collaborative module and connects them virtually to exchange network events and security rules. The CNSMS also has a huge output from operation practice, e.g., traffic data collected by multiple sources from different vantage points, operating reports and security events generated from different collaborative UTMs, etc. As such data is huge and not easy to analyze in real-time, it needs to keep them archived for further forensic analysis ${ }^{[15-18]}$.

In this paper, we evaluate a cloud-based solution in the security center for traffic data forensic analysis. The main contribution of our work is that we propose a practical solution to collect data trace and analyze these data in parallel in a cloud computing platform. We propose to use cloud storage to keep huge volume of traffic data and process it with a cloud computing platform to find the malicious attacks. As we already operate a CNSMS that has a big data output, a practical example of phishing attack forensic analysis is presented and the required computing and storage resources are investigated. We have concluded that this phishing filters functions can be effectively scaled to analyze a large volume of trace data for phishing attack detection using cloud computing. The results also show that this solution is economical for large scale forensic analysis for other attacks in traffic data.

\section{Collaborative Network Security Management System}

\subsection{System design and implementation}

CNSMS $^{[13]}$ is deployed in a multisite environment as shown in Fig. 1 and includes Beijing Capital-Info network, IDC Century-Link, an enterprise network, and a campus network to demonstrate the workability of our system. These four sites are all managed by CNSMS in the remote security center. In each site, there are several NetSecu nodes ${ }^{[14,19]}$ that take charge in different network environments to adapt to different physical links.

During the systems operation, the collaborative mechanism runs as expected to share security events and rulesets, and new rulesets are enforced on demand as instructed by the security center. Operating reports from each NetSecu node and Prober are collected and sent back to the security center. Many network security events are observed and recorded in the deployment, such as DDoS reflected attacks, spam scatter and ad hoc $\mathrm{P} 2 \mathrm{P}$ protocols, etc.

Figure 2 illustrates the whole procedure of network

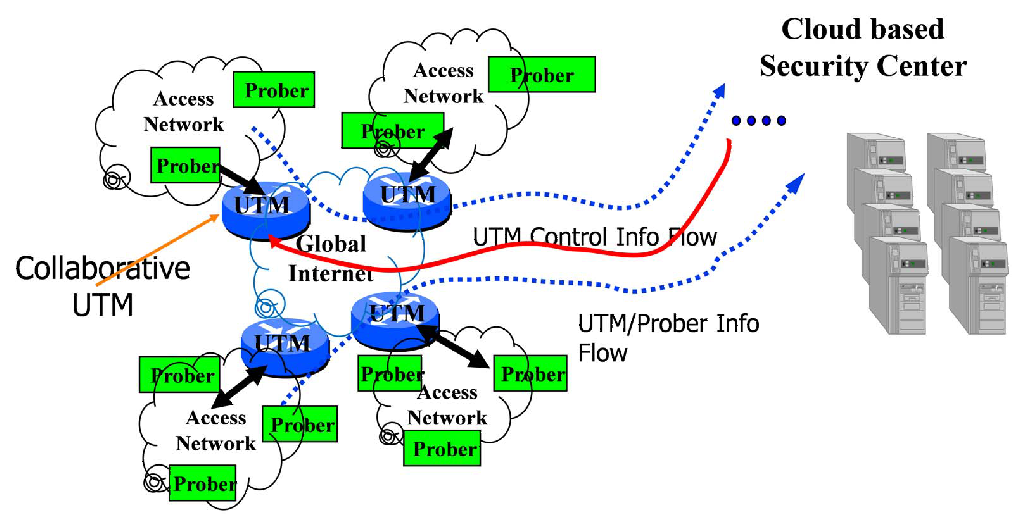

Fig. 1 Deployment of collaborative network security management system in multisite. 


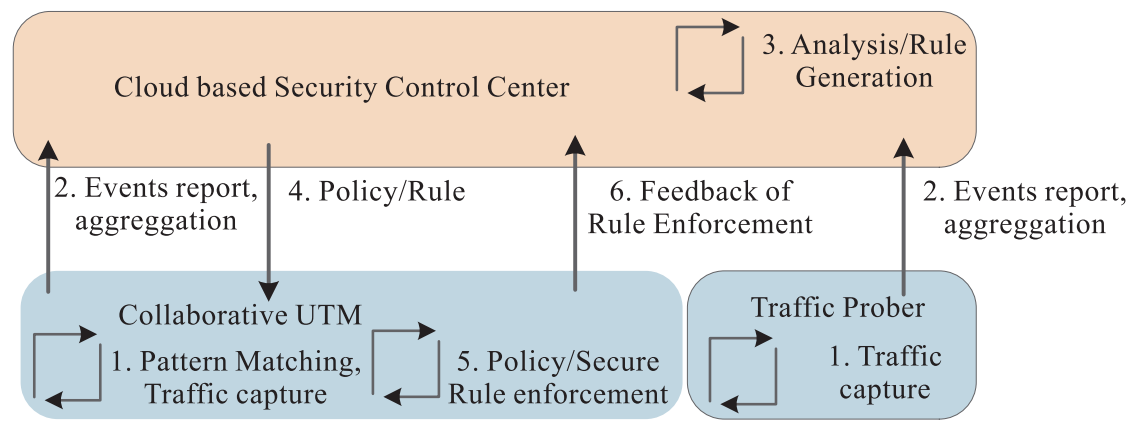

Fig. 2 Work principle of collaborative network security management system with the cloud-based security center.

security events processing. In general terms, it is an information control cycle that divides into several steps. Collaborative UTM and Probers act as sensors and report the security events and traffic data to the security center, which aggregates all the events and investigates the collected traffic data. After a detailed analysis, and with the assistance of expertise, the security center generates new policies or rulesets to disseminate to each collaborative UTM and Prober for enforcement and to receive feedback information.

\subsubsection{Traffic prober}

A traffic probe is the building block for recording the raw Internet traffic at connection level. Hyperion ${ }^{[20]}$, Time Machine ${ }^{[21,22]}$, and NProbe ${ }^{[23]}$ are all well-known representative projects in this function area. The traffic probe can be designed to focus on specific traffic occasioned by certain security events when needed.

We adapted Time Machine and deployed with $\mathrm{TIFA}^{[24,25]}$ acting as prober in either a separate device or collaborative UTM. The key strategy for efficiently recording the contents of a high volume network traffic stream comes from exploiting the heavy-tailed nature of network traffic; most network connections are quite short, with a small number of large connections (the heavy tail) accounting for the bulk of the total volume $^{[22]}$. Thus, by recording only the first $N$ bytes of each connection (the cutoff is $15 \mathrm{~KB}$ ), we can record most connections in their entirety, while still greatly reducing the volume of data we must retain. For large connections, only the beginning of a connection is recorded, as this is the most interesting part (containing protocol handshakes, authentication dialogs, data item names, etc.).

\subsubsection{Collaborative UTM}

Treated as a collaborative UTM, NetSecu is introduced in Ref. [14]. A NetSecu node consists of the following features:
(1) Incrementally deployable security elements,

(2) Can dynamically enable/disable/upgrade security functions,

(3) Policy-instructed collaboration over the Internet.

NetSecu node contains Traffic Prober, Traffic Controller, Collaborator Element, and Reporting Element to fulfill the above design goals.

A collaborator element in NetSecu manages other security elements based on the security centers command. It unites individual NetSecu platforms into a secure overlay network. The communication command between NetSecu nodes and the security center is transmitted in an SSL channel to ensure security. A collaborator can start or stop a security element at runtime and can respond to security events by, for example, limiting the DDoS traffic on demand.

NetSecu integrates security functions such as firewall, Intrusion Detection System (IPS), and AntiVirus (AV). These functions can be loaded in NetSecu nodes at runtime and can be dynamically enabled, disabled, and upgraded. Based on commodity hardware and commonly used Java with Linux, and with a mature multi-core technology, NetSecu has a comparable Maximum Loss-Free Forwarding Rate (MLFFR ${ }^{1}$ ) with bare Linux forwarding performance. Most of the security functions can run in a multi-thread model to accelerate the flow processing and pattern matching needed for UTM.

NetSecu is also equipped with bypass and selfprotection capability to resist DoS attacks in case of faults or malicious attacks occurring, to ensure high availability and survivability.

\subsubsection{Security center}

CNSMS is proposed in Ref. [13] and operated in the security center. As NetSecu nodes can manage security problems in a subdomain and provide $\mathrm{P} 2 \mathrm{P}$

${ }^{1}$ MLFFR is the highest forwarding rate with zero packet loss. 
communication interfaces ${ }^{[26]}$, CNSMS orchestrates the communication between these nodes. More specifically, CNSMS will achieve the following objectives:

(1) Security policy collaborative dissemination and enforcement,

(2) Security ruleset dissemination, enforcement, and update,

(3) Security event collaborative notification,

(4) Trust infrastructure,

(5) Scalability.

Another key function in the security center is the forensic analysis of the collected traffic and network security events. We used cloud computing in the security center to store a large volume of traffic data of different origins and conducted data analysis to generate new security rulesets as shown step 6 in Fig. 2.

To further inform the UTM how to defeat new attacks, such as a botnet, we must investigate the traffic in depth, acquire the communication graph of the botnet, and generate security rules for enforcement in the UTM to suppress the communication between bots and botmaster.

This makes it possible to resist a DDoS attack launched by a botnet. As we equipped the NetSecu node with open source application protocol identification and bandwidth management technology, the security center could instruct the system to be a collaborative distributed traffic management system, which detects and manages the traffic collaboratively after the analysis of collected traffic in the security center. It could effectively improve the identification ratio of unknown botnet protocols and throttle the DDoS traffic.

\subsection{System application-botnet suppression}

A botnet is a typical distributed attack, which is extremely versatile and is used in many attacks, such as sending huge volumes of spam or launching DDoS attacks. The work principle of a botnet is shown in Fig. 3. Suppressing botnets is increasingly difficult because the botmaster will keep their own botnets as small as possible not only to hide themselves but also to spread the botnets in an easy way. Additionally, bots can automatically change their Command and Control server $(C \& C)$ in order to hide and rescue themselves.

Based on an overlay network, Collaborative Network Security System can be used for a distributed botnets suppression system, automatically collecting network traffic from every collaborative UTM in a distributed mode and then processing these collected data in the security center. The detection algorithm proposed

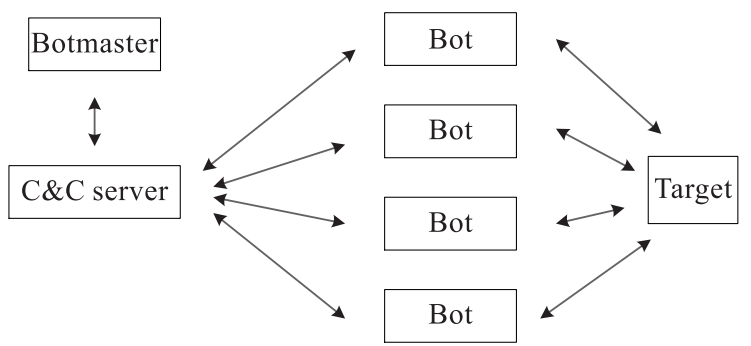

Fig. 3 Botnet structure.

by Refs. [27, 28] is based on behavior features of botnets so the system will generate and distribute rules when botnets are detected in processing. The most important feature of this system is its close loop control characteristics, i.e., gathering the feedback events resulting from the deployed rules, processing and analyzing in control nodes, removing invalid rules to make the system more efficient and reliable.

\section{Cloud-Based Forensic Analysis in Security Center}

\subsection{Cloud storage and computing platform}

We focus on traffic data storage and forensic analysis. The underground cloud storage and computing platform is based on Hadoop and Eucalyptus cloud computing. We also give some analysis of the use of cloud computing platforms based on Eucalyptus and Amazon EC2 respectively.

\subsubsection{Cloud storage with Hadoop}

The Hadoop file system ${ }^{[29,30]}$ with version 1.0 .1 is used for the cloud storage system of collected traffic. The master node is working as NameNode, SecondaryNameNode, JobTracker, Hamster, while the other nodes are working as DataNode, TaskTracker, RegionServer.

There are 4 racks of machines with 5,5,4,4 in each rack making 18 slave nodes in total. The topology is shown in Fig. 4.

The Hadoop system is used for traffic analysis whereby the traffic collected in each individual collaborative UTM is aggregated and uploaded to this cloud platform. Each node has an Intel 4 core CPU with $800 \mathrm{MHz}, 4 \mathrm{~GB}$ memory, and a $250 \mathrm{~GB}$ hard disk.

We tested the writing throughput for our Hadoop system with Hadoops TestDFSIO utility ${ }^{2}$. We also

${ }^{2}$ Hadoop TestDFSIO command: hadoop jar hadoop-test1.0.1.jar TestDFSIO -write -nrFiles 18 -fileSize 300, hadoop jar hadoop-test-1.0.1.jar TestDFSIO -write -nrFiles 36 -fileSize 100. 


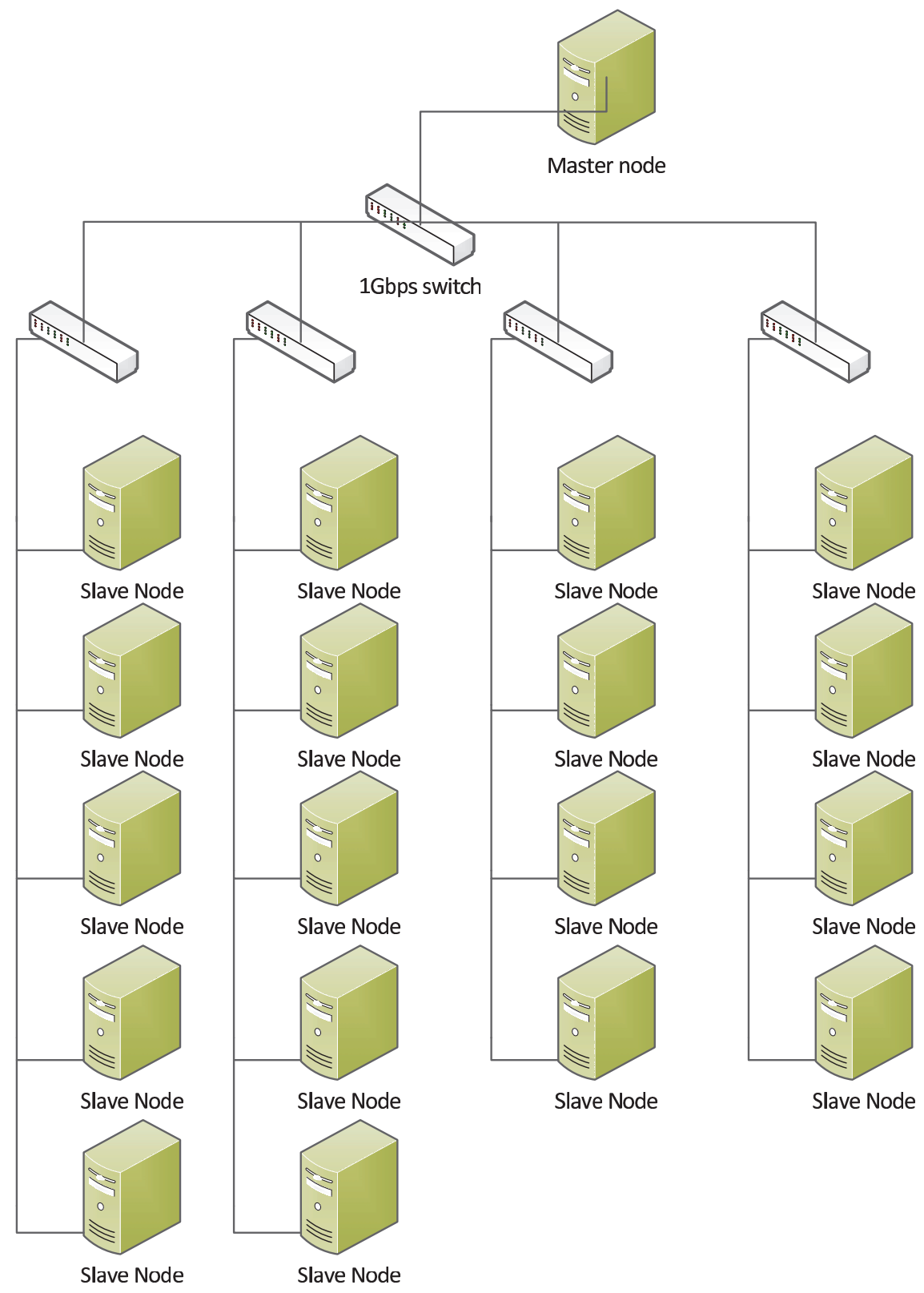

Fig. 4 Cloud storage for traffic data collected with collaborative UTM and prober.

tested two scenarios where we wrote 18 files of $300 \mathrm{MB}$ each and 36 files of $100 \mathrm{MB}$ each. The final results are shown in Table 1.

\subsubsection{Cloud computing IaaS platform}

\subsubsection{Cloud computing based on Eucalyptus}

In this section, we introduce our cloud computing platform based on Eucalyptus, an open-source platform used by NASA and Ubuntu Enterprise Cloud.

Figure 5 shows the Eucalyptus cloud computing platform we used. As shown in Fig. 5, Eucalyptus Compute consists of seven main components, with the cloud controller component representing the global state and interacting with all other components. An API Server acts as the web services front end for the cloud

Table 1 The average writing throughput of Hadoop files system in the cloud platform.

\begin{tabular}{ccc}
\hline Number of files & File size (MB) & $\begin{array}{c}\text { Total wirte } \\
\text { throughput }(\mathrm{MB} / \mathrm{s})\end{array}$ \\
\hline 95 & 100 & 215.120 \\
95 & 200 & 378.630 \\
95 & 300 & 460.055 \\
57 & 200 & 153.390 \\
57 & 100 & 324.830 \\
19 & 200 & 59.670 \\
19 & 100 & 119.190 \\
\hline
\end{tabular}




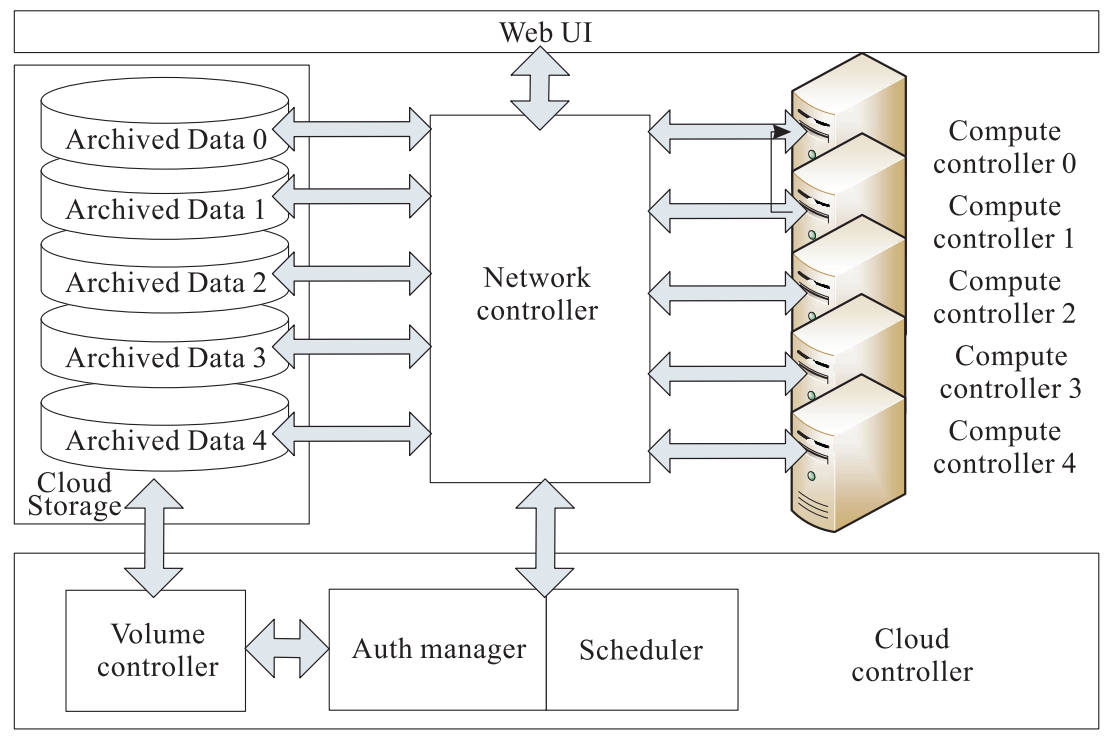

Fig. 5 Cloud computing platform based on Eucalyptus.

controller. The compute controller provides compute server resources, and the object store component provides storage services. An AuthManager provides authentication and authorization services. A volume controller provides fast and permanent block-level storage for the servers while a network controller provides virtual networks to enable servers to interact with each other and with the public network. A scheduler selects the most suitable controller to host an instance.

Our computer cluster consists of 10 heterogeneous servers. Each server is equipped with the following hardware parameters:

(a) Intel Core 2 Quad processor with $1.333 \mathrm{GHz}$ FSB and $2 \mathrm{MB}$ cache, double channel 4 GB DDR3 with $1.066 \mathrm{GHz}$, Intel G41 + ICH7R Chipset and Intel 82574L Network Chipset;

(b) Dual Intel Xeon5X00 series processors with Intel 5000P+ESB2 chipset, E5330 + 8 GB;

(c) Intel Xeon 5X00 series with FSB - 4.8/5.86/ 6.4 GT/s QPI Speed with Intel $5520+$ ICH10R chipset, $24 \mathrm{~GB}$.

In Eucalyptuss terms, there is one cloud controller and the others are nodes. The cloud controller acts as the computing portal, task assigner, and result aggregator. There is an instance affiliated with each node. In our usage scenario, we ran $4 \mathrm{VM}$ instances in each node, hence there were about 24 running instances simultaneously. Each computing instance runs the pipeline divided into the following phases: data fetcher, data processing, and posting results. Using this method, we could achieve the best working efficiency of hardware and software resources usage.

\subsubsection{Cloud computing based on Amazon}

Amazon EC2 and S3 were used for comparative analysis. The main reason for using Amazons service was to compare it to our bespoke Eucalyptus system. In consideration of user privacy and legal issues, we ensured all data was made anonymous before uploading to the Amazon S3 service.

\subsubsection{Forensic analysis of phishing attack}

Phishing is an intriguing practical problem due to the sensitive nature of stolen information (e.g., bank user account names and passwords) and is responsible for an estimated of billions of dollars loss annually. Not only users but the backing financial institutions such as e-banks and e-pay systems are impacted by phishing attacks.

There is already much research ${ }^{[31-33]}$ into phishing attack countermeasures. To protect web browser users from phishing attacks, plugins to compare visited URLs with blacklisted URLs are already provided by main-stream web browsers. Google also provides the Safe Browser API ${ }^{[3]}$ for checking an URL in Googles collected phishing database.

Some research on the life-cycle of phishing web sites is given in Ref. [2], and the results show that phishing URLs are quite ephemeral, making the collection of forensics ${ }^{[15-18,34,35]}$ difficult. Most internet users are oblivious to the dangers of phishing attacks, making combating them even harder. 
Maier et al. ${ }^{[36]}$ proposed a traffic archiving technology for post-attack analysis in Bro IDS. Using Time Machine, the network trace data is archived and can be fed back to the IDS at a later date when more current data is available to use updated forensic details of attacks. Thomas et al. ${ }^{[37]}$ proposed the Monarch system for real-time URL spam filtering for tweets and spam mail streams, whereas we put emphasis on phishing forensic analysis of large volumes of offline trace with cloud computing platforms ${ }^{[38]}$.

Similarly, we proposed an offline phishing forensic collection and analysis system. This system was targeted to solve the following challenging problems:

(1) How to collect the original data to search the phishing attack forensics therein;

(2) How to handle the huge volume of data in a reasonably short time.

A cloud computing platform ${ }^{[39-41]}$ was used for offline phishing attack forensic analysis. First, our CNSMS collected the network trace data and reported to the security center. Then we constructed an IaaS cloud platform ${ }^{[42]}$ and used existing cloud platforms such as Amazon EC2 and S3 ${ }^{[43-45]}$ for comparison. All phishing filtering operations were based on cloud computing platforms and run in parallel with a divide and conquer scheme.

\subsubsection{Data trace collection}

Our trace data was an un-interrupted collection of about six months worth of multiple vantage points deployed by the UTM. The total size of traffic passed through our vantage points was about $20 \mathrm{~TB}$. The total data was about $20 \mathrm{~TB}$ and divided into $512 \mathrm{MB}$ data blocks. Figure 6 gives a daily traffic graph from one vantage point.

Typically, as shown in Fig. 6, HTTP traffic account for most of the daily traffic. A typical $512 \mathrm{MB}$ of collected data block consists of about $40 \mathrm{~K}$ HTTP URLs. Counting the HTTP URLs visited by users, an explored URLs distribution is as shown in Fig. 7.

The experimental data was about $1 \mathrm{~TB}$ when collected in a cut-off mode in a collaborative UTM. The data trace was still growing in size during our experiments.

\subsubsection{Data anonymization}

To protect users privacy and avoid legal issues in the research, the trace data was anonymized by replacing IP and other user information before data processing in Amazon EC2.

\subsubsection{Data processing}

(1) File splitting:

Each packet capture file created by Time Machine is $512 \mathrm{MB}$, and is further divided into smaller parts for processing by using tcpdump ${ }^{[46]}$. This is due to the amount of memory used during the extraction of data from TCP streams that would exceed the maximum physical memory.

(2) TCP stream reassembly:

This stage is to restore the TCP streams in the captured pcap files using tcptrace ${ }^{[46]}$.

(3) URL extraction:

After extracting data from TCP streams, grep is used to find all URLs contained in the data by

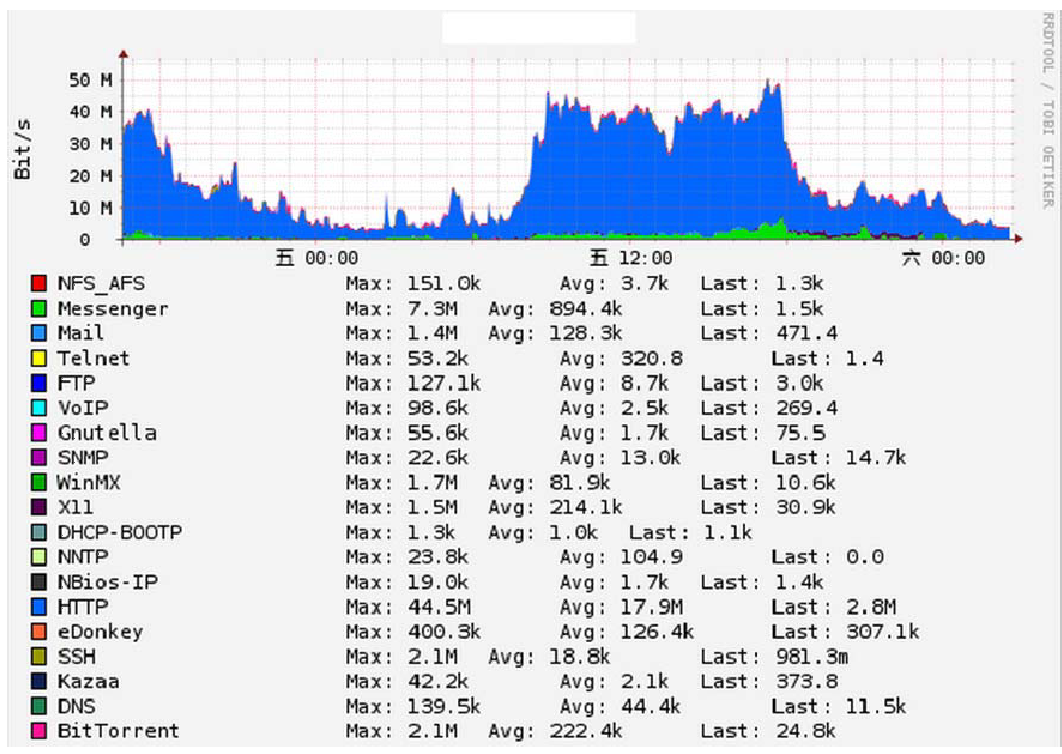

Fig. 6 Daily traffic observed and collected by Traffic Prober. 


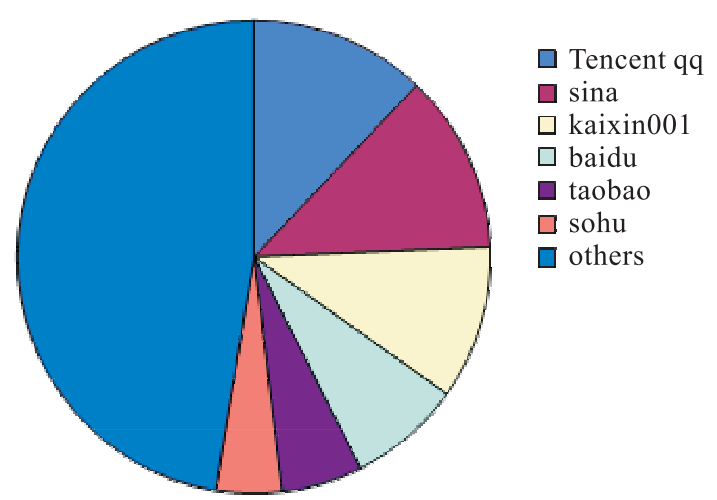

Fig. 7 HTTP URLs distribution in a typical $512 \mathrm{MB}$ trace data.

searching for lines starting with "Referer: http://."

(4) URL check:

URLs found are stored in a file to be checked for phishing by using Googles Safe Browsing $\mathrm{API}^{[3]}$. In order to check URLs for phishing sites, we use phishing URL database of Google. Google provides the first 32 bits of phishing sites' SHA256 values for users to use. If a match is found, the full 256 bits hash value is sent to Google to check the site. More details on data provided by Google can be found in Google Safe Browsing API's documentation ${ }^{[3]}$. During the process of comparing URLs' hash values, a prefix tree is used for matching because the data provided by Google is only 32 bits long and a prefix tree can do the matching of a URL's SHA256 value with Google's data in $O(1)$ time.

(5) Result reporter:

This stage collects the final results in different machines and aggregates the final report.

\subsection{Experimental results}

We conducted our evaluation experiments both on Eucalyptus and Amazon AWS for comparative purposes.

\subsubsection{Eucalyptus}

We ran the phishing data block processing task in our bespoke Eucalyptus platform with Intel Core 2 Quad Processor with $1.333 \mathrm{GHz}$ FSB and $2 \mathrm{MB}$ cache, double channel $4 \mathrm{~GB}$ DDR3 with $1.066 \mathrm{GHz}$, Intel G41+ICH7R Chipset, and Intel 82574L Network Chipset.

Times taken in different process stages in the Eucalyptus platform were measured and concluded as shown in Table 2.
Table 2 Time taken in different stages with Eucalyptus.

\begin{tabular}{lc}
\hline \multicolumn{1}{c}{ Stage } & Time (s) \\
\hline TCP stream reassembly & 1520 \\
URL extraction & 1620 \\
URL check & 5 \\
\hline
\end{tabular}

It seems the prefix tree comparisons speed is quite fast and this time spending can be almost ignored. However, before checking the URL, it takes some time to download the Google Safe Browsing signature libraries. This time spending is quite undetermined due to the network status and Google servers response latencies.

It is also pointed out that the $\mathrm{m} 1$ small instance in EC2 is memory constrained without swap partition support. It will cause problems when consuming a large volume of memory (exceeding the memory usage limit) during trace data analysis.

\subsubsection{Amazon AWS}

Trace file processing is written in Python and executed on an EC2 small instance running Ubuntu Linux 10.04. As Linuxs command shows, the host CPU is Intel(R) Xeon(R) CPU E5430 @ $2.66 \mathrm{GHz}$ with a cache size of $6 \mathrm{MB}$ and $1.7 \mathrm{~GB}$ memory (with HighTotal: $982 \mathrm{MB}$, LowTotal: $734 \mathrm{MB}$ ).

Different processing stages incur different time consumptions and are measured in Table 3. TCP stream reassembly procedures still cost most of the processing time as it needs more logic in processing.

Compared with the Eucalyptus case, it seems that the CPU used in the Amazon instance has better performance than the QX9400 quad core CPU in our physical server as shown at the URL check stage. Because of large IO operations in reassembly and extraction, the Amazon case costs much more time than the Eucalyptus case.

\subsubsection{Estimating the number of instances}

Assume the time spent in an instance to handle a $k$-byte data block in stage (2), stage (3), and stage (4) is $t_{1}, t_{2}$, and $t_{3}$ (in seconds), respectively. Assume there are $m$ collaborative UTMs or probers to collect traffic data, the average traffic throughput is $f$ during the last 24

Table 3 Time spending in different stages with Amazon EC2.

\begin{tabular}{lc}
\hline \multicolumn{1}{c}{ Stage } & Time (s) \\
\hline TCP stream reassembly & 287 \\
URL extraction & 47 \\
URL check & 12 \\
\hline
\end{tabular}


hours and the traffic cut-off factor is $h$.

The number of total instances $L$ in parallel needed to handle all of the last 24 hours traffic is calculated as follows:

$$
\begin{gathered}
T=t_{1}+t_{2}+t_{3}, \\
L=(m \times f \times T \times h) / k .
\end{gathered}
$$

$L$ is also affected by several factors such as the percentage of HTTP streams in the traffic, number of URLs in HTTP streams, the users behavior in exploring web sites, etc.

In the Eucalyptus case, we only ran one instance in each physical server. Assume $m=4, f=$ $100 \mathrm{MB} / \mathrm{s}(800 \mathrm{Mbps})$ in $1 \mathrm{Gbps}$ link, $h=0.2$ (means $20 \%$ traffic is captured), each block is $200 \mathrm{MB}$, $T=40 \mathrm{~s}$, then the number of physical servers (or instances) in parallel is calculated as follows:

$L=(m \times f \times T \times h) / k=4 \times 100 \times 40 \times 0.2 / 200=16$.

In the Amazon EC2 case, $T=330 \mathrm{~s}$, and the required number of EC2 $\mathrm{m} 1$ small instances in parallel is calculated as follows:

$L=(m \times f \times T \times h) / k=4 \times 100 \times 330 \times 0.2 / 200=132$.

\section{Conclusions}

The CNSMS is very useful to countermeasure distributed network attacks. Its operation resulted in big data outputs, such as network traffics, security events, etc. In this paper, we proposed using cloud computing systems to explore the large volume of collected data from CNSMS to track the attacking events. Traffic archiving was implemented in collaborative UTMs to collect all the network trace data and the cloud computing technology was leveraged to analyze the experimental data in parallel. An IaaS cloud platform was constructed with Eucalyptus and existing cloud platforms such as Amazon EC2 and S3 were used for comparison purposes. Phishing attack forensic analysis as a practical case was presented and the required computing and storage resource were evaluated by using real trace data. All phishing filtering operations were cloud-based and operated in parallel, and the processing procedure was evaluated. The results show that the proposed scheme is practical and can be generalized to forensic analysis of other network attacks in the future.

\section{Acknowledgements}

This work is supported by the National Key Basic Research and Development (973) Program of China
(Nos. 2011CB302805, 2011CB302505, 2012CB315801, and 2013CB228206), and the National Natural Science Foundation of China (No. 61233016). This work is also supported by Intel Research Councils UPO program with the title of Security Vulnerability Analysis Based on Cloud Platform.

\section{References}

[1] P. Knickerbocker, D. Yu, and J. Li, Humboldt: A distributed phishing disruption system, in Proc. IEEE eCrime Researchers Summit, Tacoma, USA, 2009, pp. 112.

[2] S. Sheng, B. Wardman, G. Warner, L. F. Cranor, J. Hang, and C. Zhang, An empirical analysis of phishing blacklists, in Proc. Sixth Conference on Email and AntiSpam (CEAS 2009), California, USA, 2009, pp. 1-10.

[3] Google Safe Browsing v2 API, http://code.google.com/ apis/safebrowsing/, 2012.

[4] APWG, http://www.apwg.org/ or http://www.antiphishing. org/crimeware.html, 2012.

[5] StopBadware, http://stopbadware.org/, 2012.

[6] D. Ruan, Z. Chen, J. Ni, and P. D. Urgsunan, Handling high speed traffic measurement using network processors, in Proc. 2006 International Conference on Communication Technology (ICCT 2006), Beijing, China, 2006, pp. 1-5.

[7] J. Ni, Z. Chen, C. Len, and P. Ungsunan, A fast multipattern matching algorithm for deep packet inspection on a network processor, in Proc. 2007 International Conference on Parallel Processing (ICPP 2007), 2007, Xi'an, China, pp. 16.

[8] Z. Chen, C. Lin, J. Ni, D. Ruan, B. Zheng, Z. Tan, Y. X. Jiang, X. Peng, A. Luo, B. Zhu, Y. Yue, Y. Wang, P. Ungsunan, and F. Ren, Anti-worm NPUbased parallel bloom filters in Giga-Ethernet LAN, in Proc. IEEE International Conference on Communications (ICC), Istanbul, Turkey, 2006, pp. 2118-2123.

[9] Z. Chen, C. Lin, J. Ni, D. Ruan, B. Zheng, Z. Tan, Y. Jiang, X. Peng, A. Luo, B. Zhu, Y. Yue, J. Zhuang, F. Feng, Y. Wang, and F. Ren, Anti-worm NPU-based parallel bloom filters for TCP-IP content processing in Giga-Ethernet LAN, in Proc. 1st IEEE LCN Workshop on Network Security (WoNS 2005), Sydney, Australia, 2005, pp. 748-755.

[10] R. Bye, S. A. Camtepe, and S. Albayrak, Collaborative intrusion detection framework: Characteristics, adversarial opportunities and countermeasures, in Proc. USENIX Symposium on Networked Systems Design and Implementation, Cambridge, MA, USA, 2007, pp. 1-12.

[11] F. Cuppens and A. Mige, Alert correlation in a cooperative intrusion detection framework, in Proc. IEEE Symposium on Security and Privacy, Berkeley, California, USA, 2002, pp. 205-215.

[12] A. Hofmann, I. Dedinski, B. Sick, and H. de Meer, A novelty driven approach to intrusion alert correlation based on distributed hash tables, in Proc. 2007 IEEE International Conference on Communications (ICC), Glasgow, Scotland, 2007, pp. 71-78. 
[13] B. Mu, X. Chen, and Z. Chen, A collaborative network security management system in metropolitan area network, in Proc. the 3rd International Conference on Communications and Mobile Computing (CMC), Qingdao, China, 2011, pp. 45-50.

[14] X. Chen, B. Mu, and Z. Chen, NetSecu: A collaborative network security platform for in-network security, in Proc. the 3rd International Conference on Communications and Mobile Computing (CMC), Qingdao, China, 2011, pp. 59-64.

[15] W. H. Allen, Computer forensics, IEEE Security \& Privacy, vol. 3, no. 4, pp. 59-62, 2005.

[16] M. A. Caloyannides, N. Memon, and W. Venema, Digital forensics, IEEE Security \& Privacy, vol. 7, no. 2, pp. 1617, 2009.

[17] F. Raynal, Y. Berthier, P. Biondi, and D. Kaminsky, Honeypot forensics part I: Analyzing the network, IEEE Security \& Privacy, vol. 2, no. 4, pp. 72-78, 2004.

[18] F. Raynal, Y. Berthier, P. Biondi, and D. Kaminsky, Honeypot forensics part II: Analyzing the compromised host, IEEE Security \& Privacy, vol. 2, no. 5, pp. 77-80, 2004.

[19] F. Deng, A. Luo, Y. Zhang, Z. Chen, X. Peng, X. Jiang, and D. Peng, TNC-UTM: A holistic solution to secure enterprise networks, in Proc. 9th IEEE International Conference for Young Computer Scientists(ICYCS 2008), Zhangjiajie, China, 2008, pp. 2240-2245.

[20] P. Desnoyers and P. Shenoy, Hyperion: High volume stream archival for retrospective querying, in Proc. USENIX Annual Technical Conference, Santa Clara, CA, USA, 2007, pp. 45-58.

[21] S. Kornexl, V. Paxson, H. Dreger, A. Feldmann, and R. Sommer, Building a time machine for efficient recording and retrieval of high-volume network traffic, in Proc. 2005 Internet Measurement Conference (IMC 2005), Berkeley, CA, USA, 2005, pp. 267-272.

[22] G. Maier, R. Sommer, H. Dreger, A. Feldmann, V. Paxson, and F. Schneider, Enriching network security analysis with time travel, in Proc. ACM SIGCOMM 2008, Seattle, WA, 2008, pp. 183-194.

[23] L. Deri, V. Lorenzetti, and S. Mortimer, Collection and exploration of large data monitoring sets using bitmap databases, traffic monitoring and analysis, Lecture Notes in Computer Science, vol. 6003, pp. 73-86, 2010.

[24] J. Li, S. Ding, M. Xu, F. Han, X. Guan, and Z. Chen, TIFA: Enabling real-time querying and storage of massive stream data, in Proc. 1st International Conference on Networking and Distributed Computing (ICNDC), Hangzhou, China, 2011, pp. 61-64.

[25] Z. Chen, X. Shi, L. Ruan, F. Xie, and J. Li, High speed traffic archiving system for flow granularity storage and querying, in Proc. 6th International Workshop on Performance Modeling and Evaluation of Computer and Telecommunication (ICCCN 2012 workshop on PMECT), Munich, Germany, 2012, pp. 1-5.

[26] D. Peng, W. Liu, C. Lin, Z. Chen, and X. Peng, Enhancing Tit-for-Tat strategy to cope with free-riding in unreliable
P2P networks, in Proc. 3rd IEEE International Conference on Internet and Web Applications and Services (ICIW 2008), Athens, Greece, 2008, pp. 336-341.

[27] F. Han, Z. Chen, H. Xu, and Y. Liang, A collaborative botnets suppression system based on overlay network, International Journal of Security and Networks, vol. 7, no. $4,2012$.

[28] F. Han, Z. Chen, H. Xu, and Y. Liang, Garlic: A distributed botnets suppression system, in Proc. IEEE ICDCS workshop on the First International Workshop on Network Forensics, Security and Privacy (NFSP), Macau, China, 2012, pp. 634-639.

[29] C. Lam, Hadoop in Action, Second Edition, Greenwichi: Manning Publications Co., 2012.

[30] Apache Hadoop, http://hadoop.apache.org, 2012.

[31] B. Wardman, G. Shukla, and G. Warner, Identifying vulnerable websites by analysis of common strings in phishing URLs, in Proc. IEEE eCrime Researchers Summit, Tacoma, USA, 2009, pp. 1-13.

[32] S. Li and R. Schmitz, A novel anti-phishing framework based on honeypots, in Proc. IEEE eCrime Researchers Summit, Tacoma, USA, 2009, pp. 1-13.

[33] R. Layton, P. Watters, and R. Dazeley, Automatically determining phishing campaigns using the USCAP methodology, in Proc. IEEE eCrime Researchers Summit, Dallas, USA, 2010, pp. 1-6.

[34] N. Sklavos, N. Modovyan, V. Grorodetsky, and O. Koufopavlou, Computer network security: Report from MMM-ACNS, IEEE Security \& Privacy, vol. 2, no. 1, pp. 49-52, 2004

[35] B.D. Carrier, Digital forensics works, IEEE Security \& Privacy, vol. 7, no. 2, pp. 26-29, 2009.

[36] G. Maier, R. Sommer, H. Dreger, and V. Paxson, Enriching network security analysis with time travel, in Proc. ACM Sigcomm, Seattle, WA, USA, 2008, pp. 183-194.

[37] K. Thomas, C. Grier, J. Ma, V. Paxson, and D. Song, Monarch: Providing real-time URL spam filtering as a service, in Proc. IEEE Symposium on Security and Privacy, Oakland, California, USA, 2011, pp. 447-462.

[38] T. Li, F. Han, S. Ding, and Z. Chen, LARX: Largescale anti-phishing by retrospective data-exploring based on a cloud computing platform, in Proc. 3rd Workshop on Grid and P2P Systems and Applications (GridPeer), Maui, Hawaii, 2011, pp. 1-5.

[39] L. A. Barroso, J. Dean, and U. Holzle, Web search for a planet: The google cluster architecture, IEEE Micro, vol. 23, no. 2, pp. 22-28, 2003.

[40] S. Ghemawat, H. Gobioff, and S. Leung, The google file system, in Proc. USENIX ACM Symposium on Operating Systems Principles(SOSP03), New York, USA, 2003, pp. 29-43.

[41] J. Dean and S. Ghemawat, MapReduce: Simplified data processing on large clusters, in Proc. 6th Symposium on Operating System Design and Implementation (OSDI 2004), San Francisco, California, USA, 2004, pp. 139-147.

[42] Eucalyptus, open source Cloud Computing platform, http://www.eucalyptus.com, 2012. 
[43] S. L. Garfinke, An evaluation of Amazons grid computing services: EC2, S3 and SQS, Technical Report TR-08-07, 2007.

[44] Amazon web services, Amazon elastic compute cloud

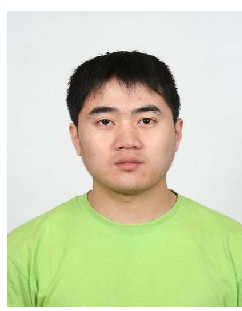

Zhen Chen is an associate professor in Research Institute of Information Technology at Tsinghua University. $\mathrm{He}$ received his BEng and $\mathrm{PhD}$ degrees from Xidian University in 1998 and 2004. He works as postdoctoral research in Network Institute of Department of Computer Science and Technology in Tsinghua University during 2004 to 2006 . He is also a visiting scholar in UC Berkeley ICSI in 2006. His research interests include overlay networking architecture, Internet security, P2P systems and Trusted Computing. He has published around 60 academic papers.

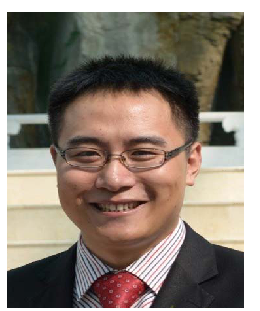

Fuye Han is a master student in Department of Computer Science and Technology in Tsinghua University. $\mathrm{He}$ graduated from PLA Information Engineering University in 2008, major in Information Engineering. His research interests include botnet, traffic archiving and other network security issues. $\mathrm{He}$ joined the Cloud Computing and IoT lab in 2010.

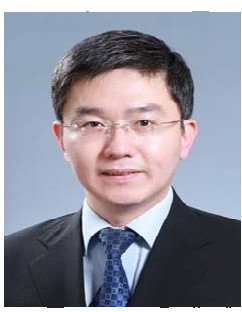

Junwei Cao is currently Professor and Deputy Director of Research Institute of Information Technology, Tsinghua University, China. He is also Director of Open Platform and Technology Division, Tsinghua National Laboratory for Information Science and Technology. His research is focused on advanced computing (amazon ec2), http://aws.amazon.com/ec2, 2012.

[45] Amazon web services, Amazon simple storage service (amazon s3), http://aws.amazon.com/s3, 2012.

[46] TCPtrace and TCPdump, http://www.tcptrace.org/ and http://www.tcpdump.org/, 2012.

technology and applications. Before joining Tsinghua in 2006, Junwei Cao was a Research Scientist of Massachusetts Institute of Technology, USA. Before that he worked as a research staff member of NEC Europe Ltd., Germany. Junwei Cao got his $\mathrm{PhD}$ in computer science from University of Warwick, UK, in 2001. He got his MEng and BEng degrees from Tsinghua University in 1998 and 1996, respectively. Junwei Cao has published over 130 academic papers and books, cited by international researchers for over 3000 times. Junwei Cao is a Senior Member of the IEEE Computer Society and a Member of the ACM and CCF

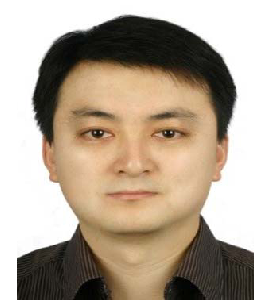

Xin Jiang is working as computer security researcher. He received the $\mathrm{PhD}$ degree in Computer Science from Institute of Computer Network of Department of Computer Science and Technology in Tsinghua University in 2010. He got BEng degree in PLA Univ. of Sci. \& Tech in 1998. His main research interests include computer network security, performance evaluation, and wireless networks.

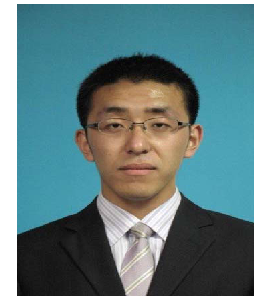

Shuo Chen is a master student supervised by Prof. Junwei Cao from Department of Automation. He got BEng degree in Tsinghua University, Beijing, China, in 2012. His research interests include distributed computing and content centric networking. 